\title{
Uma nova abordagem para o Cálculo de Balanço Hídrico Climatológico
}

\author{
Luciano Édipo Pereira da Silva ${ }^{1}$ \\ Evandro Cesar Bracht ${ }^{1}$
}

\begin{abstract}
Resumo: Este estudo visa apresentar o processo de desenvolvimento de um aplicativo para o cálculo do balanço hídrico climático. Dentre seus usos, destaca-se sua aplicação principalmente na agricultura; por isso, baseamos o seu desenvolvimento em uma plataforma para dispositivos móveis, o Android. Esta plataforma foi escolhida por ser de código aberto, gratuita e rica de recursos de desenvolvimento e execução. São descritos os procedimentos e rotinas utilizados para criar um protótipo do aplicativo.
\end{abstract}

Palavras-chave: Android. Computação móvel. Balanço hídrico.

\begin{abstract}
In this paper we present a software development process for the climatologic water balance simulation, that is used mainly in agriculture. Based in such characteristic, our development was done for a mobile devices platform, the Android. This platform was chosen because its open source and has lots of resources for development and implementation. We describe the procedures and routines that were used to create a software prototype.
\end{abstract}

Keywords: Android. Mobile computer. Water balance.

\section{Introdução}

Atualmente, no Brasil, apesar de haver uma grande rede de estações meteorológicas, que podem fornecer dados para a realização do cálculo de balanço hídrico climatológico (BHC), existe pouca oferta de aplicativos e serviços que façam o cálculo do BHC.

Balanço hídrico é a contabilização de água no solo, levando em consideração o princípio de conservação de massa, num volume de solo vegetado, ou seja, é a contabilização da entrada e saída de água no solo [11, 16].

O BHC é extremamente útil e é geralmente realizado para propriedades rurais, podendo contribuir nas tomadas de decisões do planejamento e manejo do sistema agrícola, permitindo determinar a demanda de mão de obra, tratores e implementos agrícolas; definir a época de preparo do solo, semeadura ou plantio; na aplicação de inseticidas, herbicidas, fertilizantes e corretivos; verificar a necessidade de irrigação; estimar o rendimento agrícola, eliminar ou minimizar o risco de poluição ambiental, dentre outras atividades necessárias às áreas agrícolas $[5]$.

Em áreas rurais o acesso às tecnologias como internet a cabo e adsl, é restrito, mas geralmente possuem sinal de telefonia móvel, que, juntamente com o avanço na capacidade de processamento dos celulares, possibilita a criação de software que permite acesso a dados da internet e processe equações complexas, como as que são necessárias para construção do aplicativo deste trabalho.

Dados os fatores mobilidade e conectividade oferecidos por um celular, podem-se utilizar ferramentas de desenvolvimento criadas para suportar e facilitar o uso dos recursos de hardware, como as que o Android disponibiliza para a construção de um aplicativo que possa acessar, armazenar e processar as informações meteorológicas de qualquer estação.

Baseado no sistema operacional Linux e com um ambiente de desenvolvimento flexível e poderoso, o Android é uma plataforma para dispositivos móveis que fornece ferramentas necessárias para a criação de aplicativos

\footnotetext{
${ }^{1}$ Curso de Sistemas de Informação, UEMS, Unidade de Dourados - MS 256 KM 12 - 79.840-970 - Dourados (MS) - Brasil

\{lucianoedipo, ecbracht@gmail.com\}
}

doi: $10.5335 /$ rbca.2010.002 
e utiliza a linguagem Java, com suporte a diversos serviços e hardwares, além de possuir um sistema de código aberto e livre [8].

Visando fornecer aos produtores rurais um aplicativo que realize os cálculos de BHC, foi desenvolvido o BHCMóvel para a plataforma Android. Tal aplicativo acessa a internet através da rede de telefonia; recupera dados fornecidos por estações meteorológicas, desde que estas estações estejam ligadas à internet e possuam métodos para a recuperação de seus dados; armazena os dados em banco de dados e executa os cálculos necessários para realização de uma simulação de BHC sequencial diário.

Este aplicativo se diferencia dos demais disponíveis no mercado em dois fatores:

1. Mobilidade e conectividade, fornecidas pelo uso de celulares.

2. Possibilidade de realizar o cálculo do balanço hídrico utilizando os dados pluviométricos informados pelo usuário, obtendo, assim, informações mais precisas sobre a quantidade de chuva, do local em questão (sítio, fazenda, etc.).

Inicialmente, na seção 2 são abordados alguns conceitos de BHC e suas aplicações; em seguida, na seção 3 são mostrados aspectos gerais do Android, suas características, como arquitetura, framework de desenvolvimento e aplicativo, finaliza-se com a seção 4 , sobre a definição do roteiro de cálculos utilizados no desenvolvimento do aplivativo.

\section{Balanço hídrico}

Segundo [11, 16], balanço hídrico é a contabilização de água no solo, baseado no princípio de conservação de massa em um volume de solo vegetado, ou seja, é a contabilização da entrada e saída de água no solo.

A variação de água é calculada num volume de armazenamento $\left(\Delta_{A R M}\right)$ durante um intervalo de tempo e, com esse cálculo, podem-se estimar a umidade do solo e a deficiência ou excedente hídrico.

Para o cálculo deste BHC, é necessário conhecer vários fatores, dos quais os principais são a chuva, o solo e a evapotranspiração $\left(E T_{0}\right)^{2}$. Todas essas informações podem ser obtidas pelos dados fornecidos pela maioria das estações meteorológicas. A evapotranspiração pode ser estimada por meio de dados meteorológicos, como radiação, temperatura, velocidade do vento e umidade. Neste trabalho a estimativa é realizada pelo método de Penman-Monteith FAO [1].

A entrada de água no solo ocorre principalmente por meio de chuva e irrigação. Tais valores podem ser obtidos através dos dados disponibilizados por estações meteorológicas ou pluviométricas. No caso da irrigação, considera-se a quantidade de água utilizada. A saída de água ocorre através da evapotranspiração $\left(E T_{O}\right)$, que é ajustada de acordo com a cultura e o solo, obtendo-se, assim, a evapotranspiração real $\left(E T_{R}\right)$ [9].

\subsection{Aplicações do BHC}

Considerando o alto custo do plantio, as informações disponibilizadas por um balanço hídrico são úteis nas tomadas de decisões relativas ao planejamento e manejo de sistemas agrícolas. A consulta a essas informações é recomendada para otimizar o planejamento agrícola em todas as fases que compõem um processo produtivo [10].

Para $[11,5]$, o balanço hídrico climatológico possui várias aplicações, dentre as quais se destacam:

- disponibilidade hídrica regional: caracterizar e comparar disponibilidade hídrica média do solo;

- caracterização de secas: caracterizar períodos de seca e seus efeitos na agricultura;

- zoneamento agroclimático: disponibilizar bases para estudo climático regional, podendo a região ser considerada como apta, marginal ou inapta a um determinado cultivo;

\footnotetext{
${ }^{2}$ Evapotranspiração é o processo de transporte de água na forma de vapor para a atmosfera, de uma superfície vegetada padrão.
} 
- determinação de melhores épocas de plantio: indicar, através de simulações de um grande período, qual época do ano o cultivo estará menos sujeito a restrições hídricas;

- irrigação: gerenciar a prática de irrigação com mais economia e melhora na sua eficácia.

\section{Android}

O Android ${ }^{3}$ é uma plataforma para smartphones, baseada no sistema operacional Linux; possui diversos componentes, com uma variada disponibilidade de bibliotecas e interface gráfica, além de disponibilizar ferramentas para a criação de aplicativos [8].

Criação da Open Handset Alliance, um consórcio de mais de quarenta empresas do setor de tecnologia e comunicação, liderado pelo Google Inc., o Android tem como objetivos principais [12, 8]:

1. a oportunidade de personalização das aplicações e componentes presentes em seu sistema, por ser de código aberto e gratuito;

2. a possibilidade de desenvolvimento rápido e moderno de aplicações corporativas, uma vez que sua plataforma é moderna e flexível.
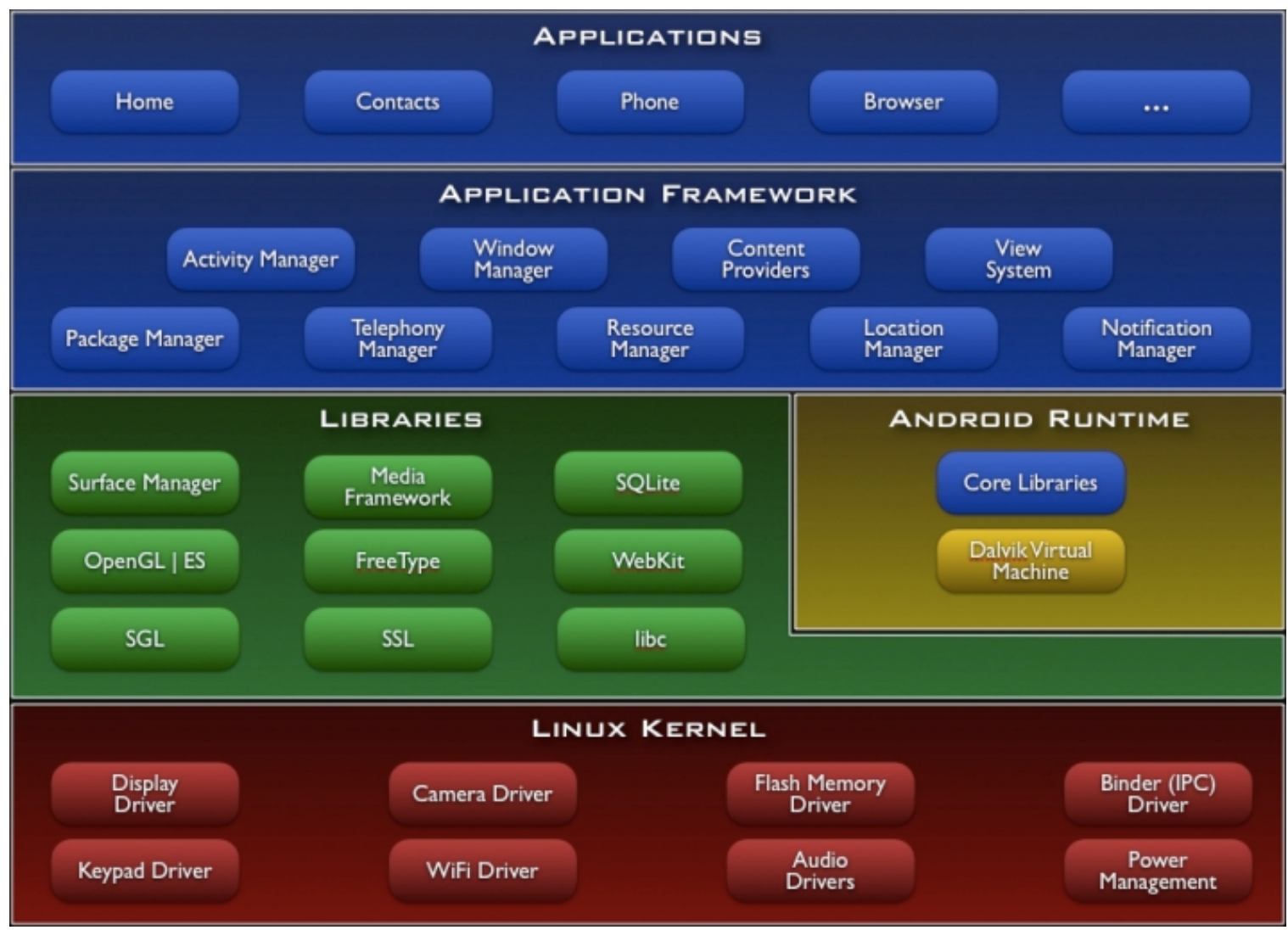

Figura 1. Arquitetura do Android [14].

\footnotetext{
${ }^{3}$ Partes desta seção são modificações do trabalho criado e compartilhado pelo Google e usado de acordo com os termos descritos na Licença Creative Commons Atribuição 2.5.
} 


\subsection{Arquitetura}

É importante conhecer a arquitetura da plataforma, seus principais componentes, bibliotecas e subsistema, para poder identificar as limitações e dependências na criação de um aplicativo para dispositivo móvel.

O Android é uma plataforma que inclui desde sistema operacional, middleware até aplicativos, e sua arquitetura é dividida em Kernel, bibliotecas, runtime, framework e aplicativos, como detalhado na Figura 1.

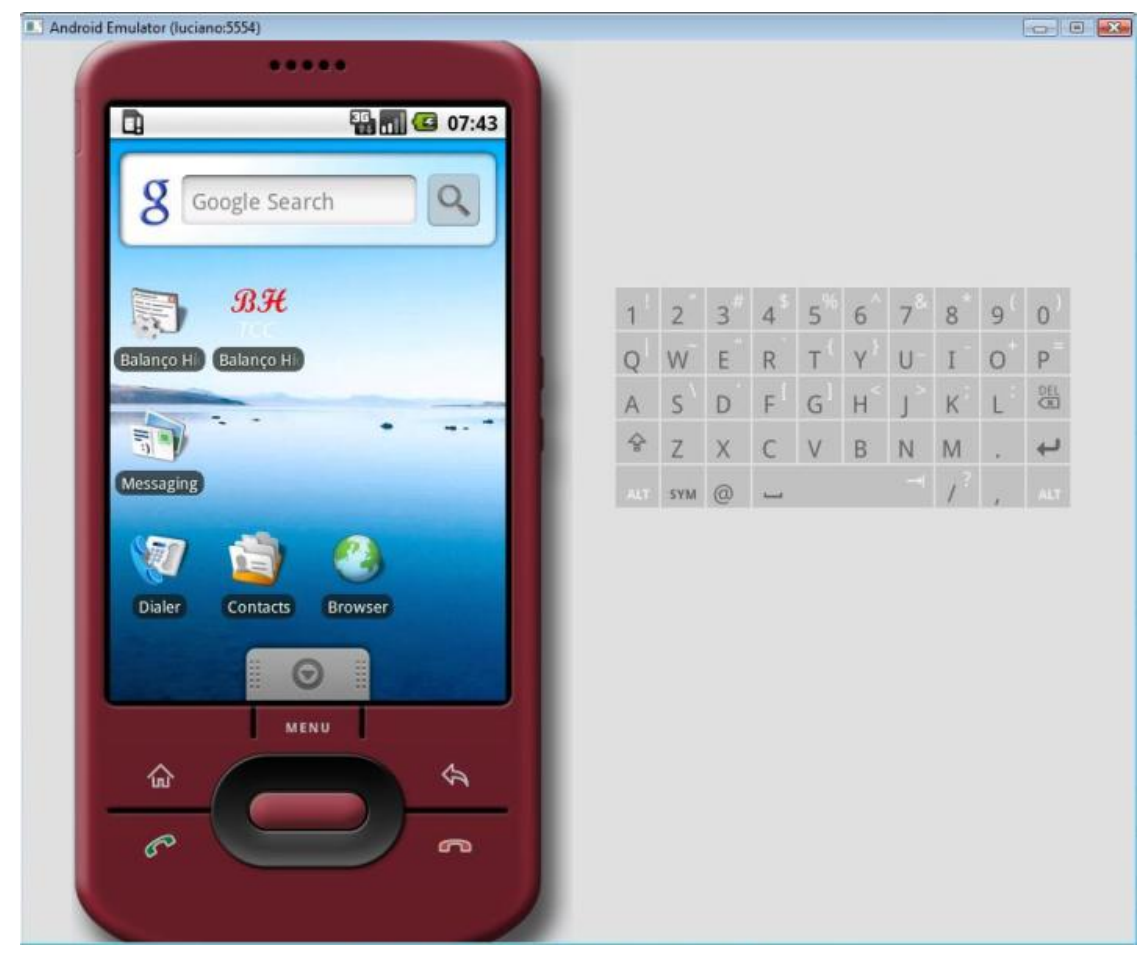

Figura 2. Tela Inicial do Emulador Android.

\subsection{Framework de Aplicativo}

O Android disponibiliza uma rica interface de desenvolvimento, oferecida por seu plugin para a IDE Eclipse e seu Emulador Android (Figura 2).

Para o desenvolvimento dos aplicativos Android, tem-se à disposição a linguagem Java, com as suas principais bibliotecas, juntamente com um conjunto de componentes prontos para uso. Os principais componentes são os gerenciadores de janela, recursos, telefone, atividade e o provedor de conteúdo [4].

\subsection{Runtime Android}

Uma aplicação Android é interpretada de maneira análoga ao byte-code Java, com a diferença de que o Android gera códigos Dalvik Executáveis (.dex) e não os byte-code (.class) do Java. Tais códigos são interpretados pela Máquina Virtual Dalvik (MVD).

Criada por Dan Bornstein, a MVD é uma alteração da Máquina Virtual Java (JVM), na qual é otimizada para os objetivos que o Android visa suprir [2].

Após a compilação, todos os arquivos .dex e outros recursos utilizados pela aplicação (Ex: imagens, sons, etc.) são compactados em um arquivo do tipo .apk (Android Package File), o qual é a aplicação finalizada e pronta para ser distribuída e instalada em qualquer dispositivo com Android [8]. 


\subsection{Framework de Desenvolvimento}

Framework de desenvolvimento, sendo um conjunto de classes, ferramentas e recursos que automatizam e auxiliam na escrita de códigos e testes, é uma estrutura construída para dar suporte à criação de aplicativos.

A construção de aplicativos Android pode ser realizada com as mesmas ferramentas utilizadas no desenvolvimento de aplicativos Java. O framework de desenvolvimento do Android oferece bibliotecas e funcionalidades específicas da plataforma para criação de aplicações móveis. O ambiente de desenvolvimento utilizado possui vários subsistemas, aplicativos e serviços, que auxiliam na construção dos aplicativos Android, como o emulador, o debugger, o plugin para IDE Eclipse, dentre outros.

\subsubsection{SDK do Android}

O Google inc. disponibilizou a primeira versão do Kit de Desenvolvimento do Android (SDK) em novembro de 2007, ainda como versão de teste, e a versão Android SDK 1.0 Release 1, em setembro de 2008. O SDK possui ferramentas que utilizamos durante a criação do aplicativo para o Android.

Durante o desenvolvimento do aplicativo ocorreram algumas atualizações no SDK do Android, a versão final do protótipo deste trabalho utiliza a versão Android SDK 1.1 Release 1, de fevereiro de 2009.

No seu desenvolvimento é utilizado o plugin para IDE Eclipse ${ }^{4}$, que é disponibilizado junto com SKD do Android, no site de desenvolvedores do Android (http://developer.android.com/sdk/1.5_r2/index.html). O plugin facilita a criação dos códigos, pois fornece recursos e interface gráfica, adiciona suporte integrado com o projeto e ferramentas Android, permitindo a construção de extensões, execução de depuração e execução do aplicativo, de forma mais rápida e fácil [13].

O emulador Android é utilizado para testar e executar as aplicações, sem a necessidade de um dispositivo físico. O emulador consegue simular tanto hardware quanto o software de um dispositivo móvel e, através do Dalvik Debug Monitor Service (DDMS), simula receber chamadas e mensagens de texto.

O emulador pode invocar outros aplicativos dentro do sistema Android, como acessar a rede do computador, executar vídeos e músicas, armazenar e recuperar dados. Possui uma variedade de debuggers e simula interrupções típicas de dispositivos móveis, tais como os recebimento de chamadas, bateria fraca, etc. Na Figura 2 pode-se ver a tela inicial do emulador.

\section{Aplicativo de Balanço Hídrico}

O Aplicativo BHCMóvel estima a água disponível no solo e fornece informações sobre a umidade do solo, água disponível para planta, deficiência hídrica e excesso hídrico, informações que podem subsidiar as tomadas de decisões na agricultura.

Os procedimentos necessários devem ser executados para a obtenção dos dados e sua correta organização no banco de dados do dispositivo móvel. Neste trabalho foram considerados três elementos principais: clima, solo e cultura.

Os dados meteorológicos necessários para a realização dos cálculos do protótipo são da região de Dourados (MS), disponibilizados pelo Sistema Agroclimático da Região de Dourados, desenvolvido pela Embrapa Agropecuária Oeste [7].

O BHCMóvel pode executar os cálculos a partir de dados de qualquer região, desde que estejam disponíveis para alimentação da aplicação. A Seção 4.2 traz a forma como os dados devem ser apresentados para que seja possível a leitura pela aplicação.

O Aplicativo possui diversas funcionalidades para a realização dos cálculos (Figura 3). Estas opções estão disponíveis já na tela inicial do aplicativo e são:

\footnotetext{
${ }^{4}$ É um ambiente integrado de desenvolvimento (IDE), de código aberto e um dos mais utilizados para programação com linguagem Java. Foi criado pela IBM e um conjunto de empresas de computação em 2001 [6]
} 


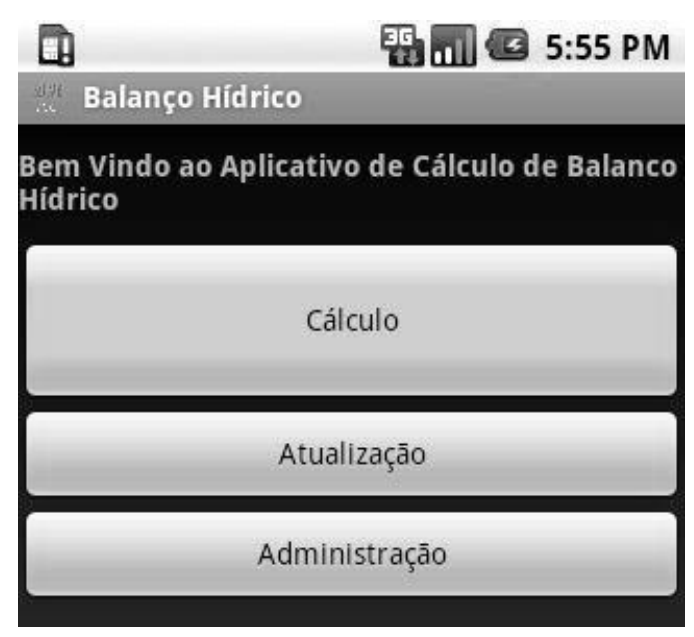

Figura 3. Tela Inicial da Aplicação.

1. Administração;

2. Coleta/Atualização;

3. Cálculo.

Existem diversas outras ações disponíveis para o usuário realizar. A Figura 4 representa cada uma dessas ações através de um diagrama de casos de uso simplificado.

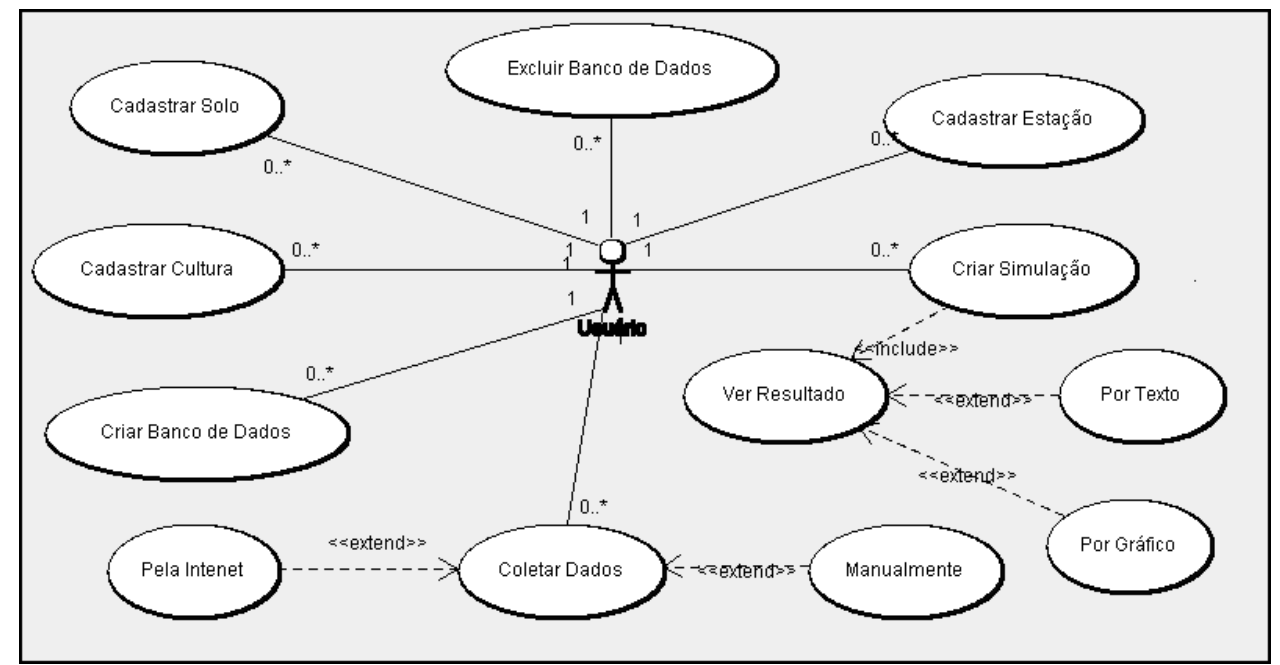

Figura 4. Diagrama de casos de uso para aplicativo de BHC

\subsection{Administração}

A administração é a parte do aplicativo que define as configurações do aplicativo, cadastro das informações de solo e cultura, além da criação do banco de dados climáticos (caso o usuário deseje utilizar mais de uma fonte).

O cadastro de solo é realizado nessa parte do aplicativo. Nesta tela encontram-se os campos para inserção dos dados de solo, como nome, espessura, capacidade de campo, ponto de murcha e densidade (Figura 5B). Quando cadastrado, o solo é inserido no banco de dados, que se encontra selecionado no início do aplicativo. 


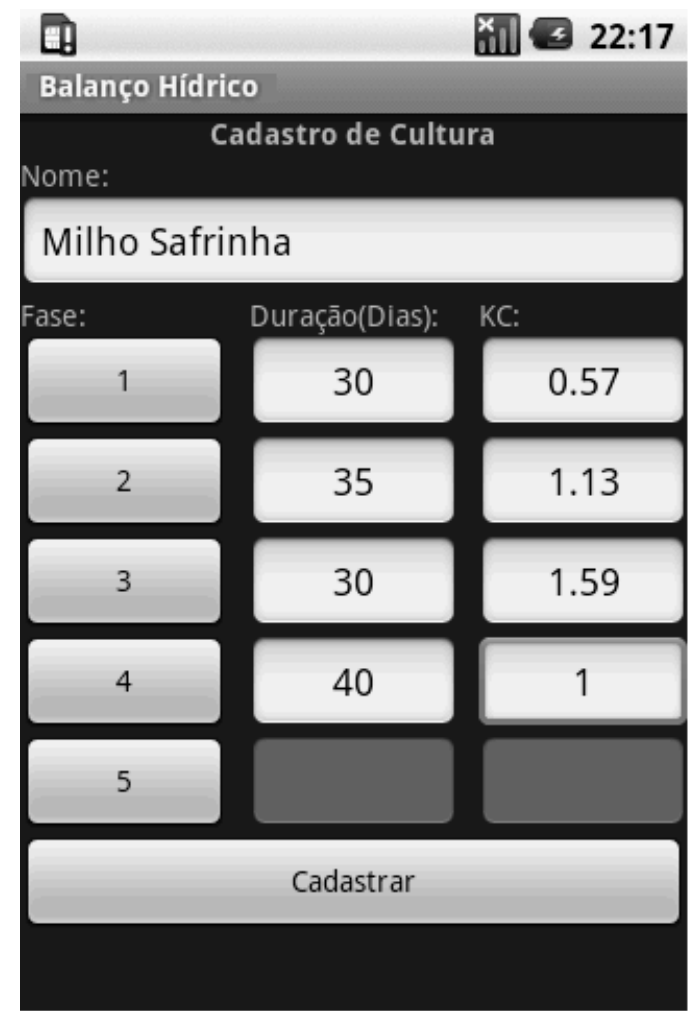

A

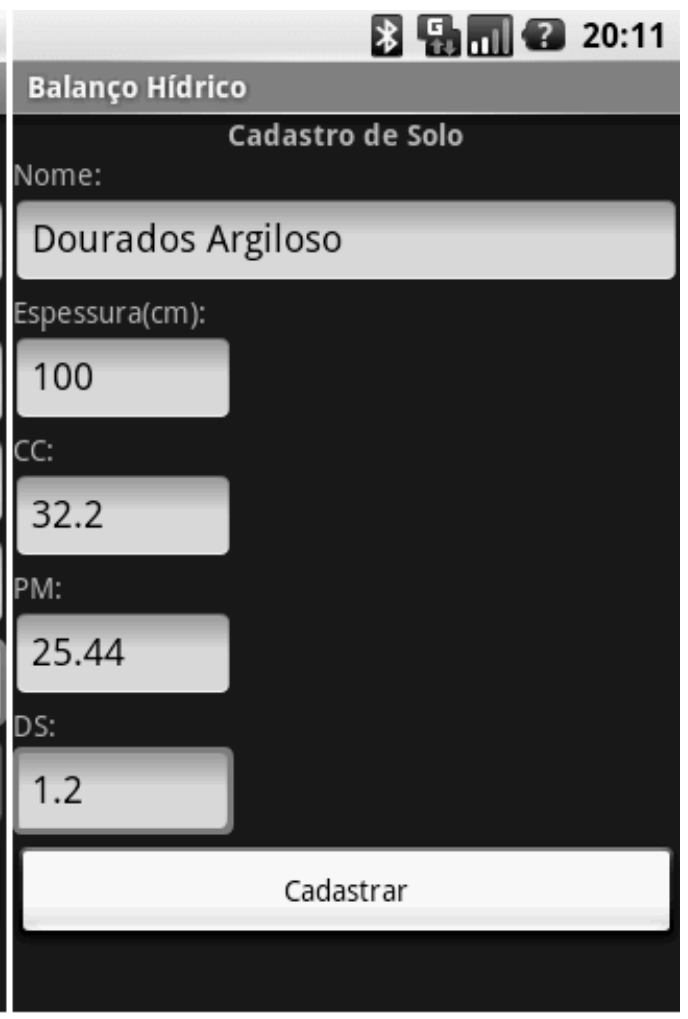

B

Figura 5. (A)Tela de cadastro de cultura, (B) Tela de cadastro do solo.

O cadastramento das culturas (Figura 5A) é executado da mesma forma que o cadastro de solo: existe uma tela para a inserção das informações de nome de cultura, fases e sua duração e qual o coeficiente de cultura $K_{C}$ na fase.

\subsection{Coleta dos Dados}

Nesta função é realizada a coleta dos dados de clima. Para o cálculo faz-se necessário inserir os dados de temperatura média diária, umidade média relativa do ar, velocidade média do vento a 2 metros, radiação líquida e precipitação. Todos esses dados, excluindo a precipitação, são necessários para calcular a evapotranspiração pelo método de Penman-Monteith-FAO [3].

Além desses dados é preciso ter informações de solo e cultura, que estão armazenados no banco de dados do aplicativo, sendo gerenciado na função Administração.

Os dados de chuva de uma estação podem não representar a ocorrência real de precipitação em áreas localizadas. Visando aproximar-se da quantidade real de chuva na área em que se deseja realizar o cálculo, foram desenvolvidas duas opções de captação dos dados de chuva: a primeira é a coleta através da internet, onde todos os dados climáticos são transferidos; a segunda é a quantidade de chuva local inserida manualmente.

A inserção manual de quantidade de chuva só deverá ser realizada se o dia em questão estiver inserido e já possua as outras informações climáticas. Entretanto, independentemente da opção de atualização dos dados de chuva, ambos são armazenados no banco de dados e podem ser utilizados durante a realização dos cálculos.

\subsubsection{Pela Internet}

$\mathrm{Na}$ atualização pela internet, são disponibilizados dois parâmetros opcionais, sendo as datas iniciais e finais. Se o período for determinado, busca-se o endereço web da estação meteorológica, previamente cadastrado no banco 
de dados e que está selecionado para o cálculo. Em seguida, é construído o link de busca, com os parâmetros adicionados. Sendo os parâmetros nulos, o link é construído com a data inicial, igual à última data encontrada nos dados já armazenados, e a data final a do dia atual.

Para alimentação do banco de dados utilizam-se os recursos que a plataforma Android disponibiliza para a importação dos dados, através da API XML/DOM. Para efeito de testes, neste trabalho os dados do Sistema Agroclimático de Região de Dourados desenvolvido pela Embrapa Agropecuária Oeste de Dourados foram utilizados.

O cadastro do endereço do Webservice ${ }^{5}$ contém as tags $\{$ DataInicial $\}$, que corresponde ao dia de ínicio da coleta de dados, e DataFinal, ao dia final para coleta de dados, que são substituídos pelas datas que o usuário informou. As informações climáticas são coletadas para cada dia do intervalo estipulado e o arquivo deve obedecer ao formato, conforme o exemplo a seguir:

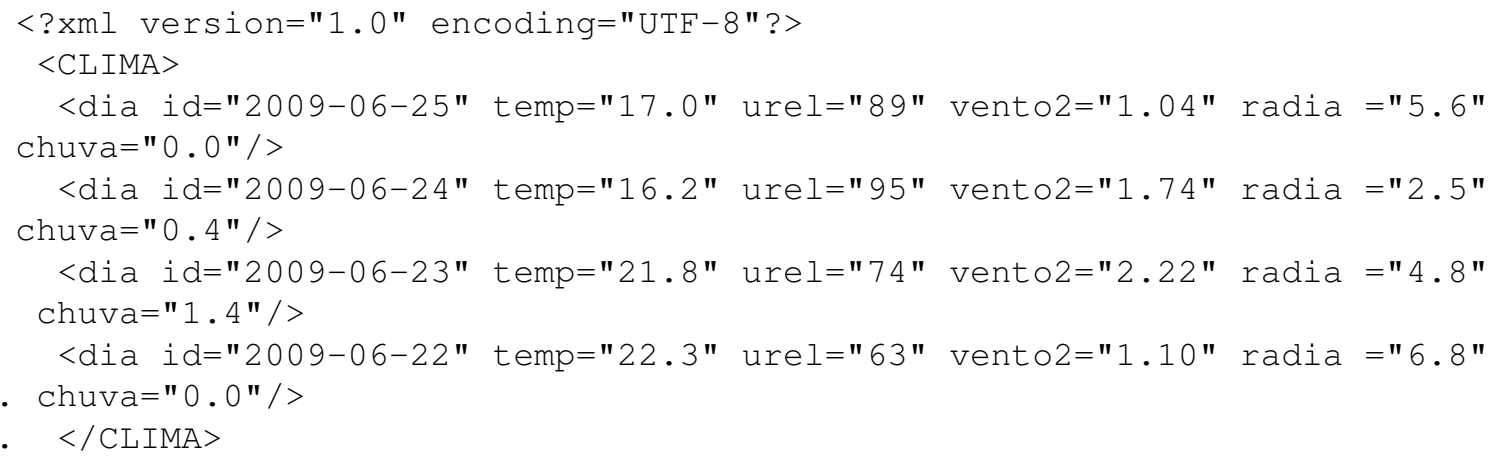

O aplicativo "lê" os dados, utilizando um parser da classe org.w3c.dom.Document, através do endereço construído. A busca fornece os dias e os dados climáticos. O aplicativo percorre cada dia, validando e inserindo na tabela climática da aplicação. A Figura 6 apresenta uma tela do aplicativo após a inserção dos dados no aplicativo.

\subsubsection{Inserção Manual}

A inserção manual é realizada por meio de uma tela que possui apenas dois campos de entrada, dia e chuva. Os dados locais são inseridos na mesma tabela climática onde se encontram os outros dados baixados da internet. Isso é necessário porque o usuário pode optar por fazer um cálculo utilizando os dados inseridos manualmente, caso estejam disponíveis, ou usando os dados obtidos da estação meteorológica.

\subsection{Cálculo}

Para calcular o balanço hídrico o aplicativo prepara os dados e realiza o roteiro que será descrito nesta subseção. Existem diversos métodos distintos para elaboração do BHC, conforme $[1,11,16]$.

Na primeira tela (Figura 7) são listadas todas as culturas inseridas no banco de dados do aplicativo. Após a cultura ser selecionada é apresentada uma tela, onde o usuário escolhe o período, a data inicial obrigatória e a data final opcional. Para facilitar a escolha das datas, é apresentado o período de dados climáticos cadastrado no banco de dados.

Em seguida deve-se escolher o solo (Figura 7). Tais dados são necessários para calcular o $C T A$ (capacidade total de água no solo em $\mathrm{mm}$ de água), a qual é calculada apenas uma vez para cada simulação, sendo realizada de acordo com a seguinte equação:

$$
C T A=\frac{C C-P M}{10} \times D s \times Z
$$

em que:

$C C$ - capacidade de campo $(\mathrm{mm})$;

$P M$ - ponto de murcha $(\mathrm{mm})$;

\footnotetext{
${ }^{5}$ Por exemplo, nos teste do foi usado o endereço: http://www.cpao.embrapa.br/clima/android/index2.php?ini= $\{$ DataInicial $\} \&$ fim $=\{$ DataFinal $\}$
} 


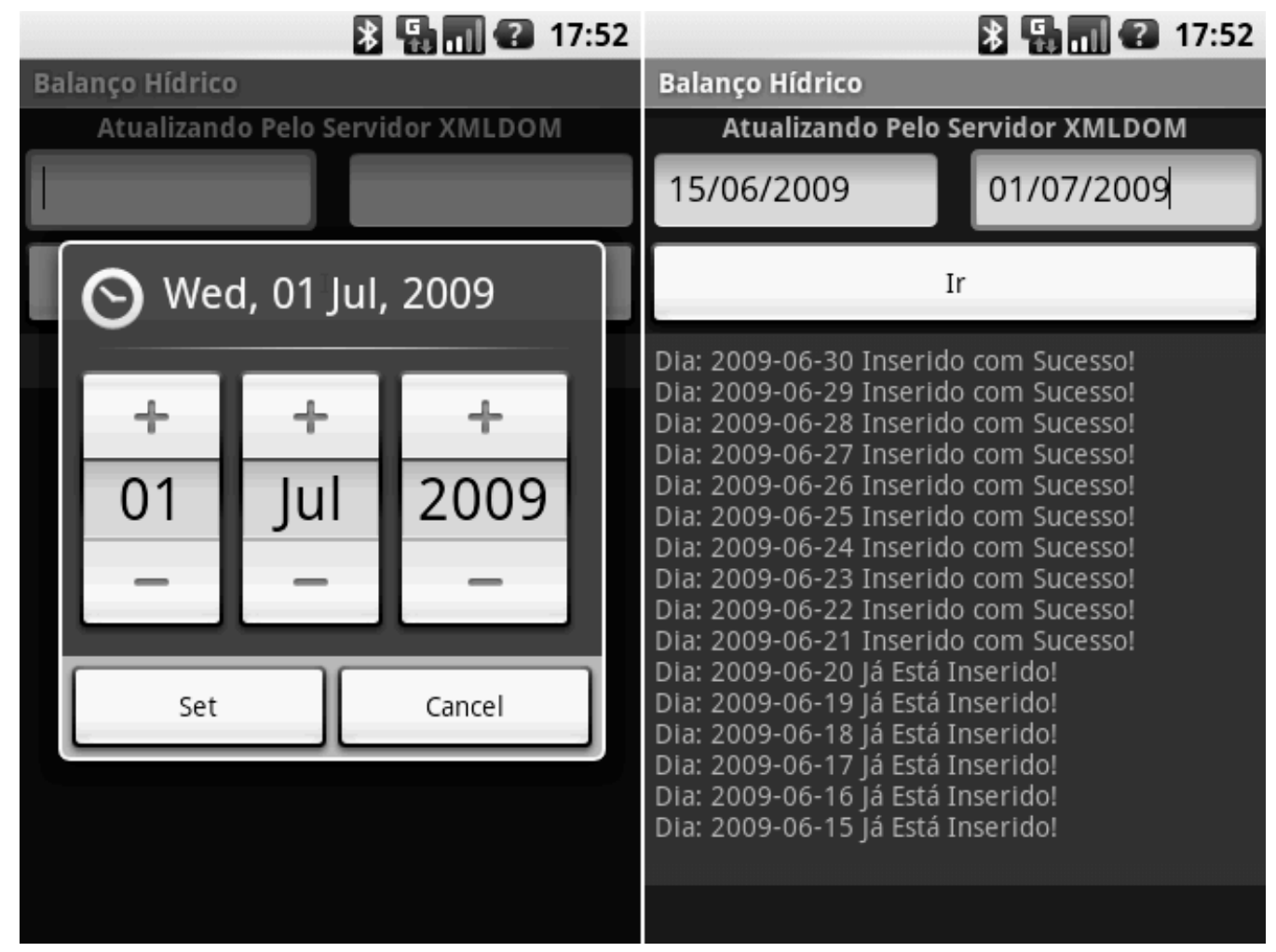

Figura 6. Telas do aplicativo para coleta de dados

$D s$ - densidade do solo;

$Z$ - espessura $(\mathrm{cm})$.

Após essas seleções e o cálculo do $C T A$, os demais cálculos, apresentados abaixo, são executados em sequência, do primeiro ao último dia, para todos os dias do intervalo selecionado.

O primeiro cálculo a ser realizado é o da evapotranspiração de referência $E T_{0}$, realizado de acordo com a equação[1]:

$$
E t_{0}=\frac{0.408 \Delta R_{n}+\gamma \frac{900}{T+273} u_{2}\left(e_{s}-e_{a}\right)}{\Delta+\gamma\left(1+0.34 u_{2}\right)}
$$

onde:

$E T_{o}$ - evapotranspiração de referência $\left(m m d i a^{-1}\right)$;

$R_{n}$ - saldo de radiação líquida $\left(M J \mathrm{~m}^{-2} \mathrm{dia}^{-1}\right)$;

$T$ - temperatura média do dia $\left({ }^{\circ} C\right)$;

$u_{2}$ - velocidade do vento a 2 metros de altura $\left(m s^{-1}\right)$;

$e s$ - pressão do vapor de saturação $(k P a)$;

$e a$ - pressão atual de vapor $(k P a)$;

$\Delta$ - curva do vapor de pressão $\left(k P a{ }^{\circ} C^{-1}\right)$;

$\gamma$ - constante psicrométrica ${ }^{6}\left(\mathrm{kPa}^{\circ} \mathrm{C}^{-1}\right)$.

Nem toda chuva ocorrida num único dia é totalmente absorvida pelo solo. Para se calcular a chuva efetiva, é realizado o cálculo da precipitação efetiva $(P e)$. Tal operação somente é necessária nos dias em que a quantidade de chuva diária exceder $25 \mathrm{ml}$, significando que apenas um percentual da quantidade de chuva foi absorvido pelo solo. Existem dois métodos principais para se calcular o Pe [15]: estimada pelo método Número Curva e Escorrimento Superficial. Este aplicativo utiliza o método Escorrimento Superficial.

\footnotetext{
${ }^{6}$ Representa o balanço entre o calor sensível ganho pela passagem de fluxo de ar no termômetro de bulbo úmido e o calor sensível transformado
} em calor latente. 


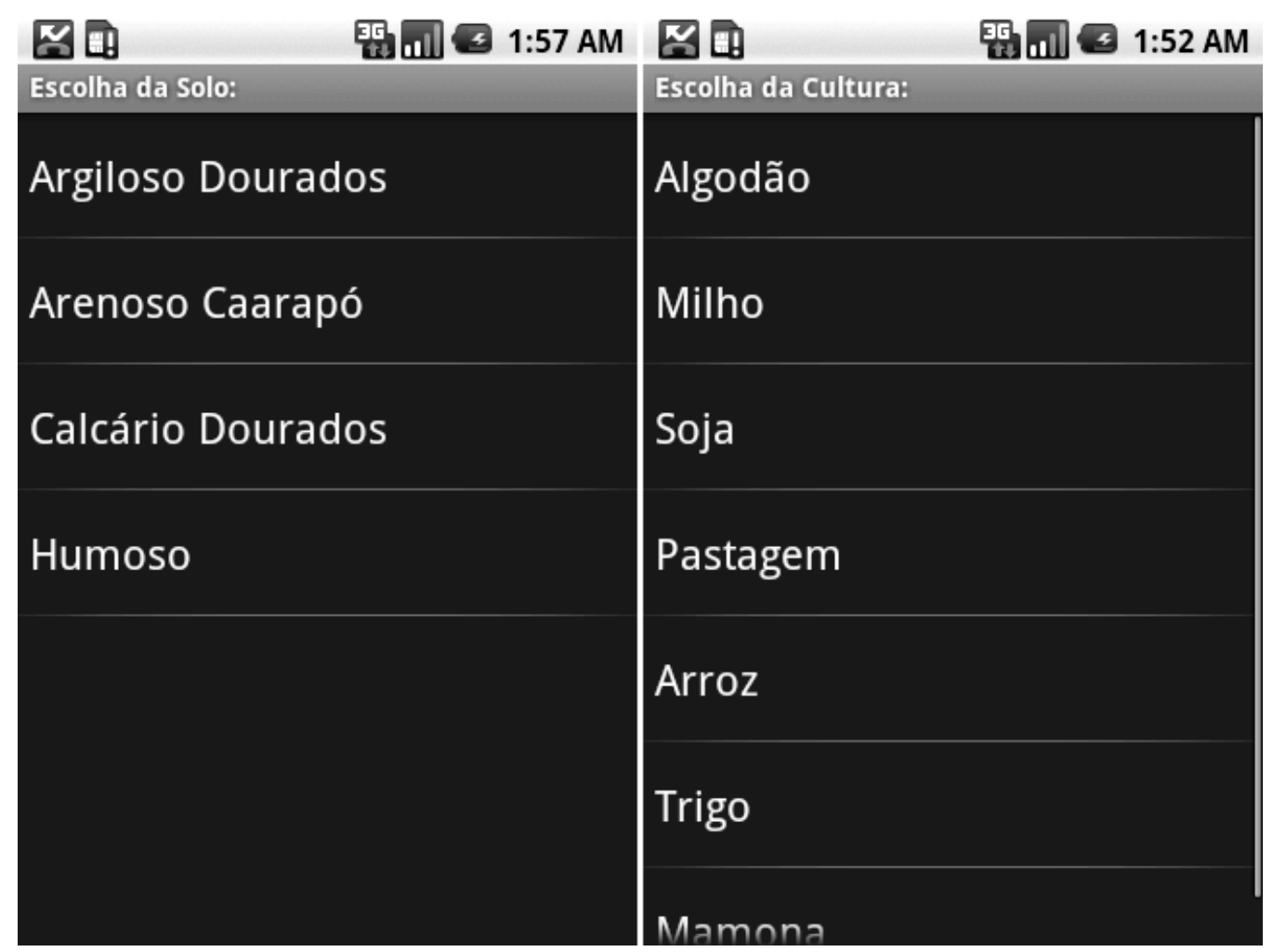

Figura 7. Tela de seleção do solo e da cultura

O cálculo do coeficiente de ajuste do solo $K_{s i}$ do dia será usado para o ajuste da $E T_{R}$ em função do teor de umidade atual do solo, sendo realizado de acordo com:

$$
K_{s i}=\frac{U_{i-1}-P M}{C C-P M}
$$

em que:

$U_{i-1}$ - umidade do solo no dia anterior;

$P M$ - ponto de murcha do solo;

$C C$ - capacidade de campo do solo.

Como referido na seção 2, a evapotranspiração de referência é estimada para uma superfície vegetada padrão (gramado), mas cada cultura possui a sua necessidade de água, o que pode também interferir na evapotranspiração. Dada essa situação, existe um fator de correção, chamado "coeficiente de cultura $K_{C}$ ", que é diferente em cada fase de evolução da cultura.

Os dados de cultura serão necessários, portanto, para saber quantas fases e quais os coeficientes de ajuste serão aplicados nas fases. No caso do balanço hídrico normal (gramado), o $K_{C}$ sempre será 1, existindo apenas uma fase.

A partir deste ponto tem-se definido o $E T_{0}, K_{s}$ e o $K_{C}$ e pode-se, então, estimar a evapotranspiração real $E T_{r}$, fazendo:

$$
E T_{r}=E T_{0} \times K_{S} \times K_{C}
$$

Com o valor de $E T_{R}$ far-se-á, em seguida, o cálculo da quantidade de água disponível no dia $\left(A D_{i}\right)$, que é a soma da água disponível do dia anterior $\left(A D_{i-1}\right)$, com a precipitação efetiva $(P e)$ menos a evapotranspiração real do dia $\left(E T_{R i}\right)$, expresso por:

$$
A D_{i}=A D_{i-1}+P e_{i}-E T_{R i}
$$




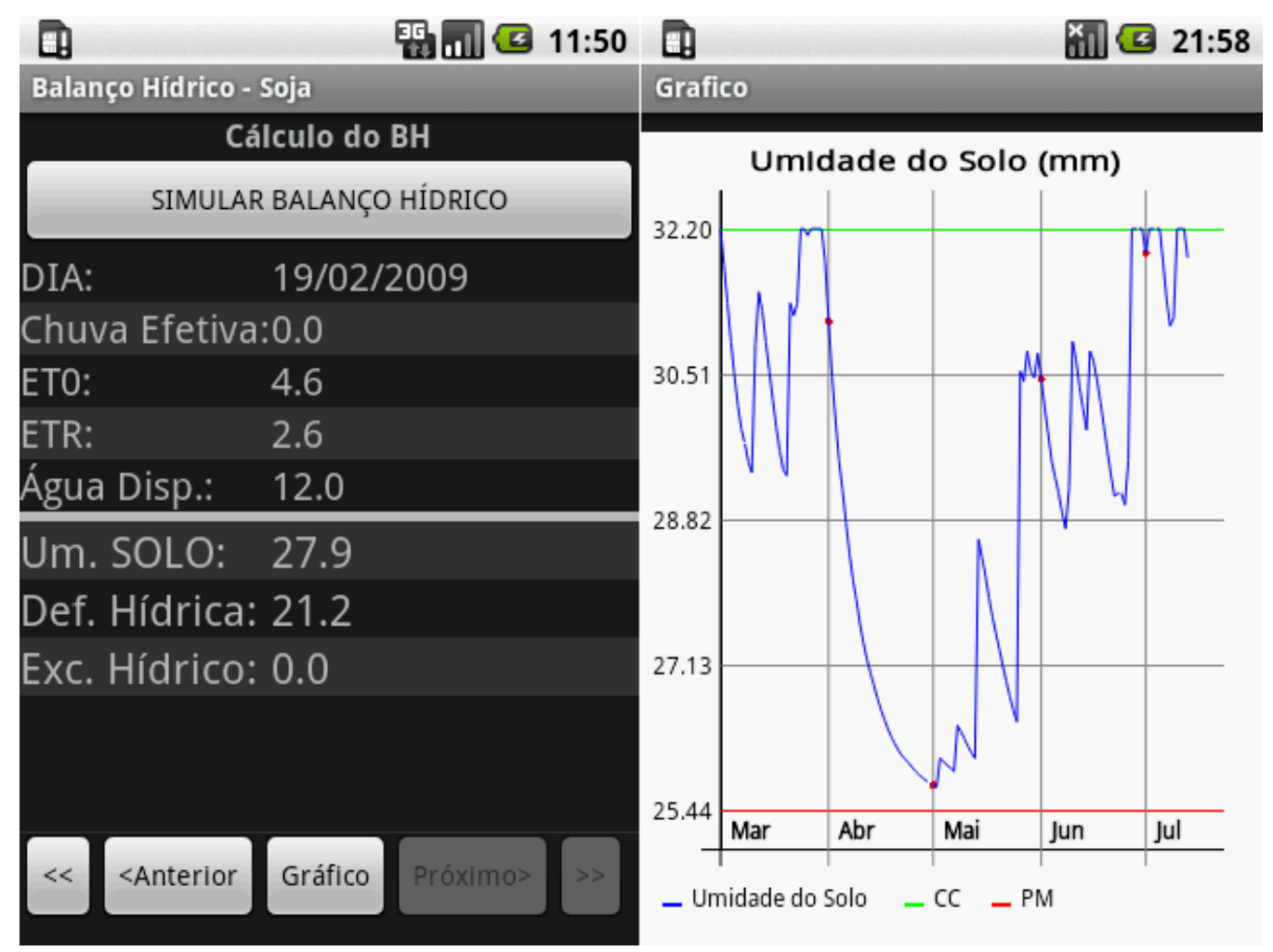

Figura 8. Ex. Telas de resultado de uma simulação

A umidade do solo é calculada de acordo com a relação entre a quantidade de água disponível e a quantidade de água que o solo poderia armazenar. A equação da umidade do solo é descrita por:

$$
U_{i}=10 \frac{A D_{i}}{D s \times Z}+P M
$$

A deficiência hídrica do solo no dia $\left(D H_{i}\right)$ é a quantidade de água que falta no solo. Para que esta atinja sua capacidade máxima de armazenamento a deficiência é calculada por:

$$
D H_{i}=C T A_{\text {solo }}-A D_{i}
$$

Finalmente, o último cálculo do balanço hídrico é o do excesso hídrico do dia $\left(E H_{i}\right)$. O excesso é a quantidade de água que o solo não pode absorver por já estar em sua capacidade máxima, sendo o excedente hídrico definido por:

$$
E H_{i}=P e-E T_{R}+\Delta A D
$$

onde $\triangle A D$ é a variação de água disponível no solo $\left(A D_{i}-A D_{i-1}\right)$.

Após executar todos os cálculos, o resultado é apresentado numa tela, com o detalhamento dos valores do último dia do cálculo, com destaque para os elementos mais importantes: a quantidade de água disponível, a umidade do solo, a deficiência e o excedente hídrico. O usuário pode percorrer os resultados para cada dia, por meio dos botões de anterior e próximo. Quando selecionados, estes atualizam os valores dos elementos na mesma tela, conforme exemplo a ser apresentado na Figura 8. O usuário possui a possibilidade de verificar os resultados de cada um dos quatro elementos, de forma gráfica.

\section{Validação}

Com o objetivo de validar os resultados obtidos no BHCMóvel, foram realizadas duas etapas: 


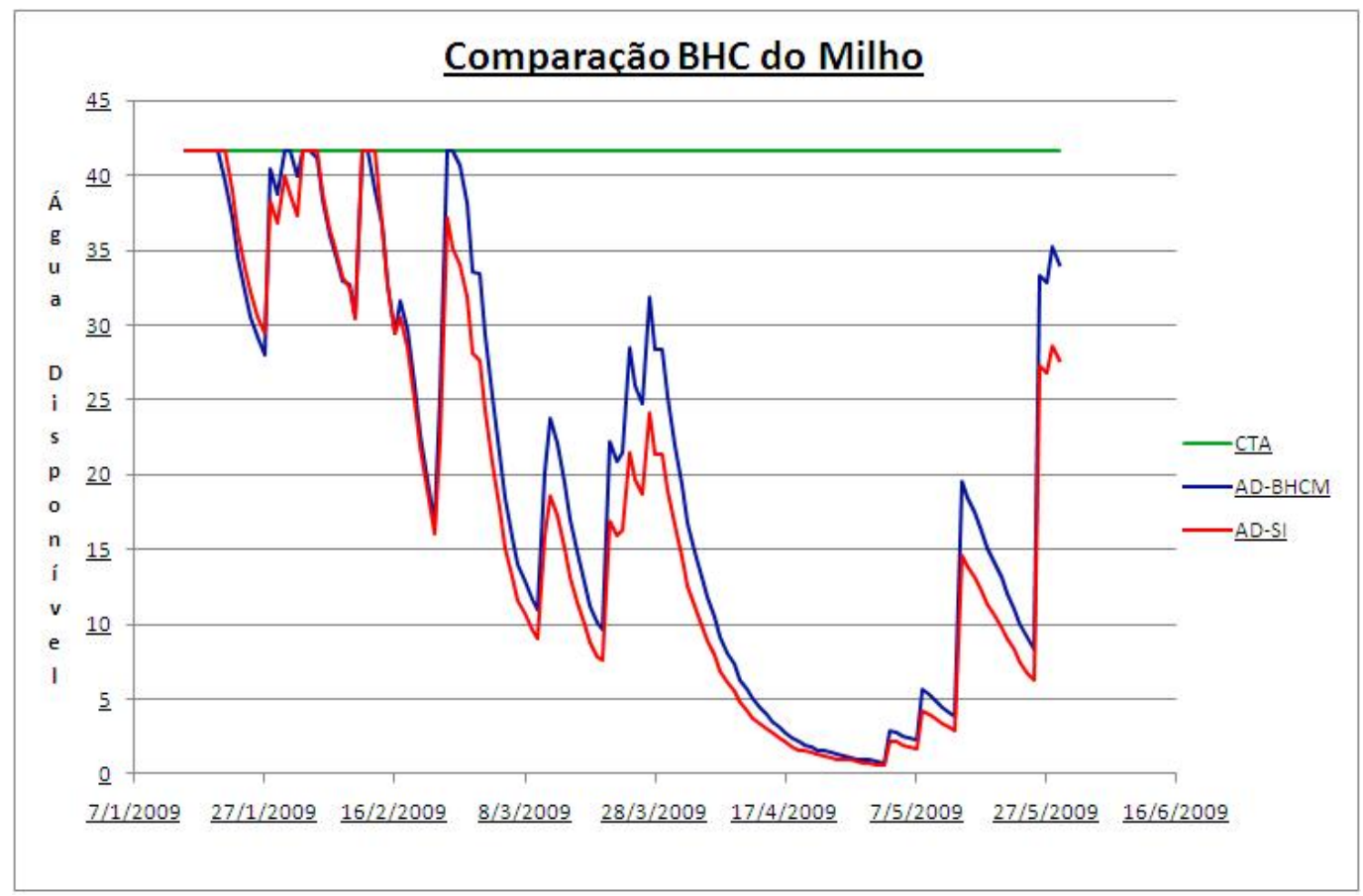

Figura 9. Comparação da água disponível para milho.

- na primeira, realizou-se uma comparação dos valores obtidos no BHCMóvel com os resultados de um segundo aplicativo (seção 5.1);

- na segunda, verificou-se a validade de calcular o BHC utilizando a quantidade de chuva de chuva informada pelo usuário, ao invés de utilizar a quantidade disponibilizada na estação.

\subsection{Aplicativo}

Com o objetivo de validar os resultados do BHCMóvel, realizamos o cálculo do BHC utilizando o Sistema de Informação Agroclimático de Dourados/MS (SIA Dourados) ${ }^{7}$, que tem como principais diferenças do BHCMóvel:

1. Para cálculo de precipitação efetiva o SIA Dourados utiliza o método Número Curva [15], ao passo que o BHCMóvel utiliza o de Escorrimento Superficial, ver seção 4.3.

2. O SIA Dourados mantém os valores de água disponível ao máximo, durante dois dias, sempre que estes chegam à capacidade total de armazenamento (CTA).

Os resultados obtidos pelo SIA Dourados (AD-SI) e BHCMóvel (AD-BHCM) podem ser vistos nos gráficos das Figuras 9 e 10. No gráfico da Figura 9 é realizado o cálculo utilizando-se os dados do período de 15/1/2009 a 29/5/2009 para uma cultura de milho, ao passo que o gráfico da Figura 10 considera os dados de 6/11/2008 a 24/2/2009 para uma cultura de soja.

Nota-se que para ambos os sistemas os valores da quantidade de água disponível no solo seguem a mesma tendência. Contudo, existem valores divergentes, como resultado das diferenças citadas acima.

\footnotetext{
${ }^{7}$ http://www.cpao.embrapa.br/clima/index.php?pg=balanco_hidrico
} 


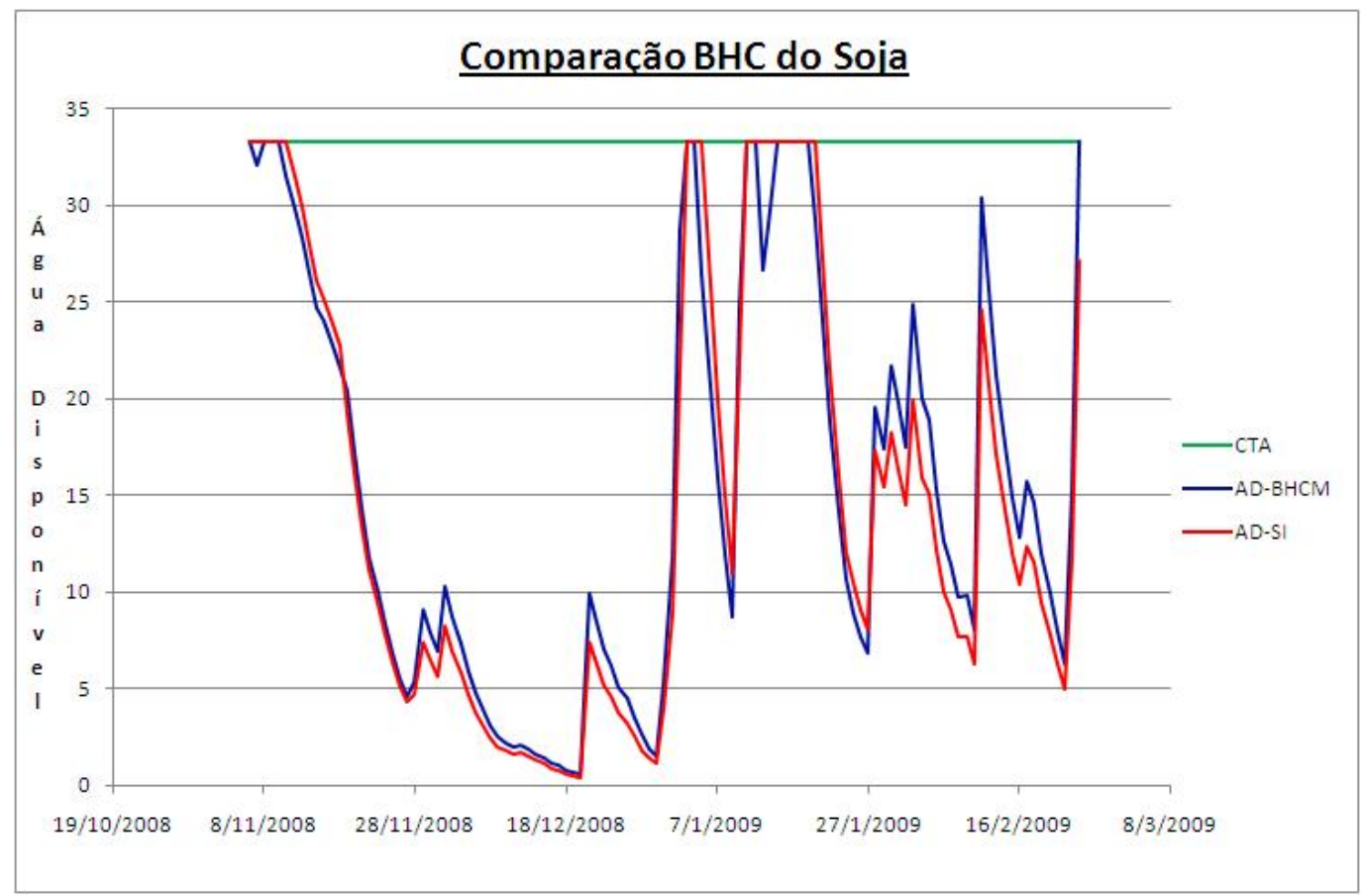

Figura 10. Comparação da água disponível para soja

\subsection{Quantidade de chuva}

Um dos diferenciais do BHCMóvel é a possibilidade de o usuário informar manualmente a quantidade de chuva para uma determinada região. Com o objetivo de verificar se tal abordagem é valida, realizaram-se cálculos com os dados de duas estações distintas, distantes $13 \mathrm{~km}$ uma da outra:

1. Estação Embrapa (estação localizada na Embrapa Agropecuária Oeste, Posição Lat. 22º 16’30S, Long. $54^{\circ} 49^{\prime} 00 \mathrm{~W}$ e altitude $\left.408 \mathrm{~m}\right)$;

2. Estação INMET (estação do Instituto Nacional de Meteorologia, Lat. 22º 19,39S, Long. 54º1'14W e altitude de $469 \mathrm{~m})$.

Tais cálculos (Figura 11) possuem as seguintes características:

- ADEMBRAPA: cálculo do BHC utilizando apenas dados da estação Embrapa;

- ADINMET: cálculo do BHC utilizando apenas dados da estação INMET;

- COMPARAÇÃO: cálculo do BHC utilizando todos os dados da estação Embrapa, exceto a quantidade de chuva, a qual é utilizada da estação INMET.

O gráfico da Figura 11 mostra que, utilizando a quantidade de chuva de uma determinada região, próxima à estação meteorológica principal ${ }^{8}$, chega-se a um resultado mais condizente com a realidade do que utilizando apenas a quantidade de chuva disponibilizada pela estação principal.

\footnotetext{
${ }^{8}$ Estação cadastrada no BHCMóvel, a qual é utilizada para se obter os dados.
} 


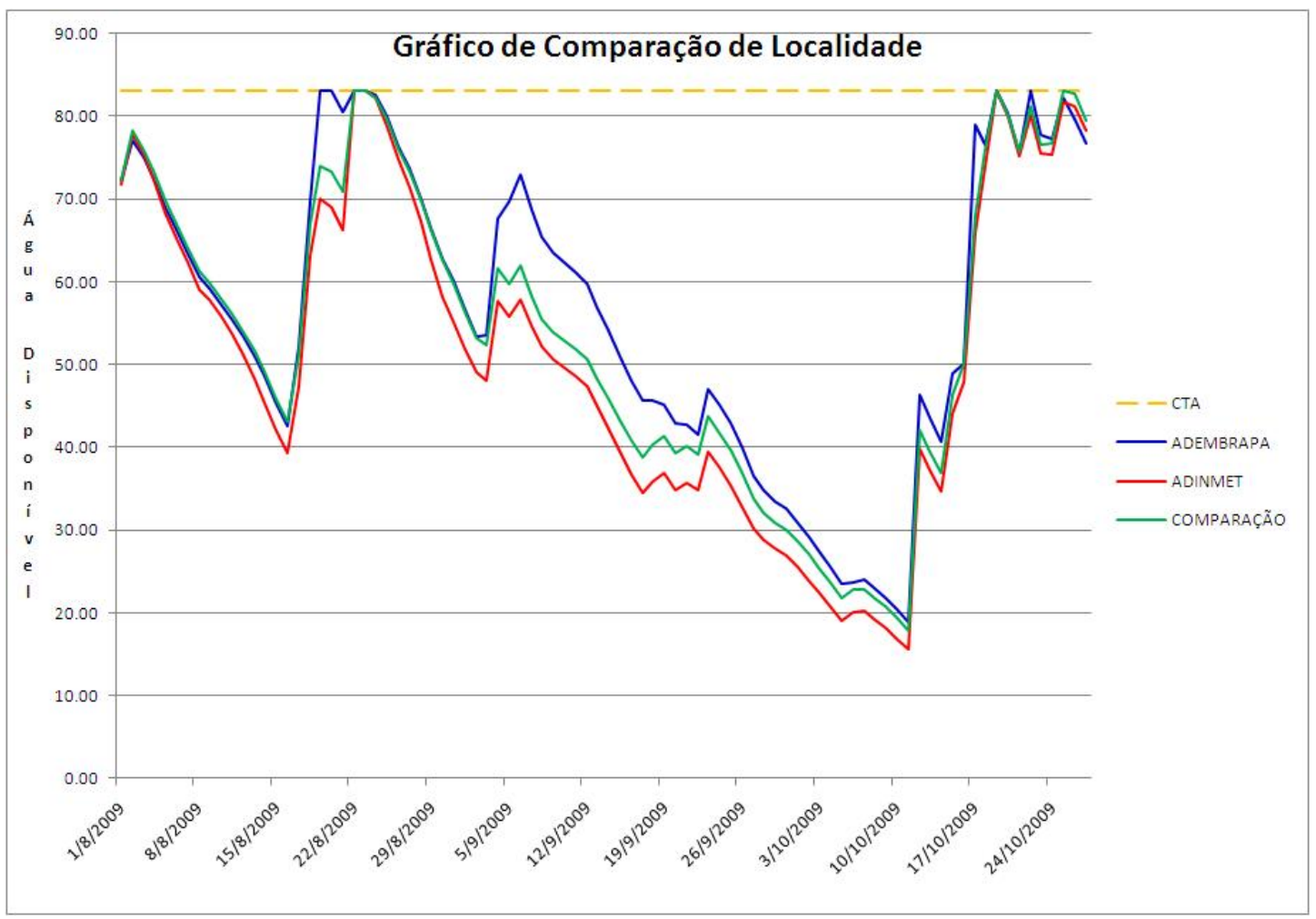

Figura 11. Comparação do resultado do BHC com dados intercalados

\section{Conclusão}

Em geral, os produtores rurais estão distantes das áreas urbanas, sendo difícil ter acesso a informações atualizadas sobre a quantidade de água disponível no solo, a qual permite um melhor planejamento e organização da propriedade rural.

Este artigo apresenta uma nova abordagem para a realização do cálculo do balanço hídrico climatológico. Tal abordagem, desenvolvida para a plataforma Android, faz uso da mobilidade e conectividade disponíveis em celulares para permitir que os usuários realizem o cálculo do balanço hídrico climatológico em qualquer lugar, além de possibilitar a entrada de dados pluviométricos específicos para uma localidade (fazenda, sítio, etc.).

Dentre as principais contribuições destaca-se ainda que este trabalho pode servir como modelo para a implementação de outros métodos de cálculo de BHC, bastando apenas alterar o roteiro dos cálculos. Pode assim como este roteiro, ser reutilizado em qualquer outra plataforma, desde que utilize linguagem Java.

\section{Agradecimentos}

Gostaríamos de agradecer à Embrapa Agropecuária Oeste que, na figura do Dr. Carlos Ricardo Fietz, possibilitou a disponibilização dos dados necessários para que este trabalho fosse desenvolvido.

\section{Referências}

[1] ALLEN, R. G. et al. Crop evapotranspiration - guidelines for computing crop water requirements FAO irrigation and drainage paper 56. Relatório Técnico, FAO - Food and Agriculture Organization of united Nations, 1998. 
[2] BORNSTEIN, D. Dalvik virtual machine (online), 2008. Disponível em: <http://www.dalvikvm.com/>. Acesso em: 11 out. 2008.

[3] CONCEIÇÃO, M. A. F. Roteiro de cálculo da evapotranspiração de referência pelo método de Penman-Monteith-FAO (online), 2006. Empresa Brasileira de Pesquisa Agropecuária. Disponível em: $<$ http://www.repdigital.cnptia.embrapa.br/handle/CNPUV/8815>. Acesso em: 20 jun. 2009.

[4] DIMARZIO, J. Android: A Programmer's Guide. McGraw-Hill Professional, ilustrada edition, 2008.

[5] DOURADO NETO, D. Balanço hídrico cíclico e sequencial: estimativa de armazenamento de água no solo. In: CONGRESSO BRASILEIRO DE IRRIGAÇÃO E DRENAGEM, XI. Campinas, Brasil, 1996. Anais... p. $30-42$.

[6] ECLIPSE FOUNDATION, I. About the eclipse foundation, 2008. Disponível em: <http://www.eclipse.org/org/>. Acesso em: 11 out. 2008.

[7] FIETZ, C. R. et al. Sistema de monitoramento agroclimático da região de Dourados, MS. In: CONGRESSO BRASILEIRO DE AGROINFORMÁTICA - SBIAGRO 2009, Viçosa, Brasil. Anais... 2009.

[8] LECHETA, R. R. Google Android - aprenda a criar aplicações para dispositivos móveis com o Android SDK. Editora Novatec, 2009.

[9] LIMA, J. E. F. W.; SILVA, C. L.; OLIVEIRA, C. A. S. Comparação da evapotranspiração real simulada e observada em uma bacia hidrográfica em condições naturais de cerrado. Revista Brasileira de Engenharia Agrícola e Ambiental, v. 5, n. 1 p. 33-41, 2001.

[10] MOTA, F. S. Meteorologia agrícola. Editora Nobel, 1986. 376 p.

[11] PEREIRA, A. R.; ANGELUCCI, L. R.; SENTELHAS, P. C. Agrometeorologia: fundamentos e aplicações práticas. Editora Agropecuária, 2002.

[12] PROJECT, A. O. S. Android open source, 2008. Disponível em: <http://source.android.com>. Acesso em: 18 jun. 2009.

[13] PROJECT, A. O. S. Android - an open handset alliance project, 2009a. Disponível em: $<$ http://developer.android.com/sdk/android-1.5.html>. Acesso em; 18 jun. 2009.

[14] PROJECT, A. O. S. What is android?, 2009b. Disponível em: <http://developer.android.com/guide/basics/what-is-android.html>. Acesso em: 15 jun. 2009.

[15] PRUSKI, F. F.; GRIEBELER, N. P.; SILVA. D. D. Comparação entre dois métodos para a determinação do volume de escoamento superficial. Revista Brasileira de Ciência do Solo, v. 25, n. 2, p. 403-410.

[16] SEDIYAMA, G. C. (1996). Estimativa da evapotranspiração: histórico, evolução e análise crítica. Revista Brasileira de Agrometeorologia, v. 4, n. 1 p. i-xii. 